\title{
A CASE REPORT OF A CADASIL PATIENT WITH SUSPECTED PARKINSON DISEASE
}

Elena Muiño ${ }^{1}$ Natividad Raña ${ }^{2}$, Ana Rodríguez-Campello ${ }^{3}$ Irene Navalpotro-Gómez ${ }^{4}$ Miguel López-Cuina ${ }^{5}$ lolanda Riba-LLena ${ }^{6}$ Natalia Cullell1 ${ }^{1}$ Caty

Carrera $^{6}$, Nuria P Torres-Aguila ${ }^{1}$, Jara Cárcel' ${ }^{1}$, Jonathan González-Sánchez ${ }^{1,7}$, Jurek Krupinski ${ }^{8}$, Joan Montanere, 9 , Jaume Roquer ${ }^{3}$, Israel Fernández-

Cadenasi 1 , 10
1. Stroke Pharmacogenomics and Genetics. Fundació Docennciai Recerca MútuaTerrassa, Hospital Mútua de Terrassa. Universitat de Barcelona; 2. Neurology Department. CHUAC; 3 . Neurology Department. IMIM-Hospital del Mar; 4 .

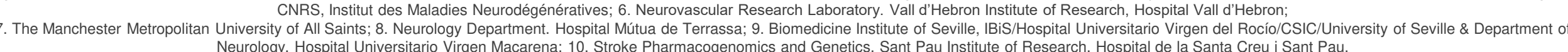

\section{Background}

CADASIL ("Cerebral Autosomal Dominant Arteriopathy with Subcortical Infarcts and Leukoencephalopathy") is an autosomal dominant inherited small-vessel disease caused by mutations in the NOTCH3 gene (19p13).

The main symptoms of CADASIL are migraine, psychiatric disorders, recurrent small subcortical infarctions and dementia; parkinsonism has been rarely reported ${ }^{1-3}$. Magnetic resonance imaging (MRI) and histopathology show small subcortical infarctions and confluent white matter hyperintensities (WMH), mostly in periventricular locations with involvement of the anterior temporal pole and the external capsule.

Parkinson's Disease (PD) is clinically characterized by rigidity, rest tremor and bradykinesia as defined by the "UK PD Society Brain Bank Clinical Diagnostic Criteria"; tipically with an unilateral onset and good response to levodopa. DaT-scan shows an asymmetric disturbance on the nigroestriatal dopaminergic system and the myocardial 123|-metaiodobenzylguanidine (MIBG) scintigraphy a significantly lower heart-tomediastinum ratio ( $\mathrm{H} / \mathrm{M}$ ratio). On the other hand, vascular parkinsonism (VP) is characterized by symmetric symptomatology, postural inestability and rare presence of rest tremor ${ }^{2}$. Usually with normal DaT-scan and MIBG scintigraphy and poor response to levodopa treatment. See table 1.

\begin{tabular}{|l|c|c|}
\hline & PD & VD \\
\hline Symptoms & Asymmetric & Symmetric \\
\hline Rest tremor & Usually & Rare \\
\hline DaT-scan & Altered & Normal \\
\hline MIBG & Altered & Normal \\
\hline Response to levodopa & Good & Poor \\
\hline
\end{tabular}

Table 1. Main difference between PD and VD.

We report the case of a CADASIL patient with suspected Parkinson Disease instead of vascular parkinsonism.

\section{Case report}

A 66 year-old female with speech disturbances was diagnosed of CADASIL disease with a p.R607C mutation in NOTCH3 gene at age of 59 .

With 64 years old she presented an important lose of weight and fatigue, reason why a thoraco-abdominal CT scan and a colonoscopy were performed, discarding a tumoral cause.

With 65 years old she came to the consultation because of a moneycounting rest tremor. She showed an asymmetric parkinsonism at the examination with a right upper-limb rest tremor, a "no-no" head tremor, bradykinesia and hypomimia, with an Unified Parkinson's Disease Rating Scale (UPDRS) part III 15/68 and a Hoehn and Yard of 2.

Her MRI showed confluent WMH, mostly in periventricular locations with involvement of the external capsule, without strategic infarcts (see figure 1). The DaT-scan evidenced an asymmetric presynaptic nigroestriatal dopaminergic degeneration with left predominance (see figure 2). The myocardial MIBG scintigraphy was not concluent as the values obteined were in the lower limit of normality (see figure 3).

Levodopa and dopaminergic agonist treatments were established with a successful response. Rest tremor was almost disappeared (UPDRS part III $8 / 68$ ) and non-motor symptoms as fatigue were improved.
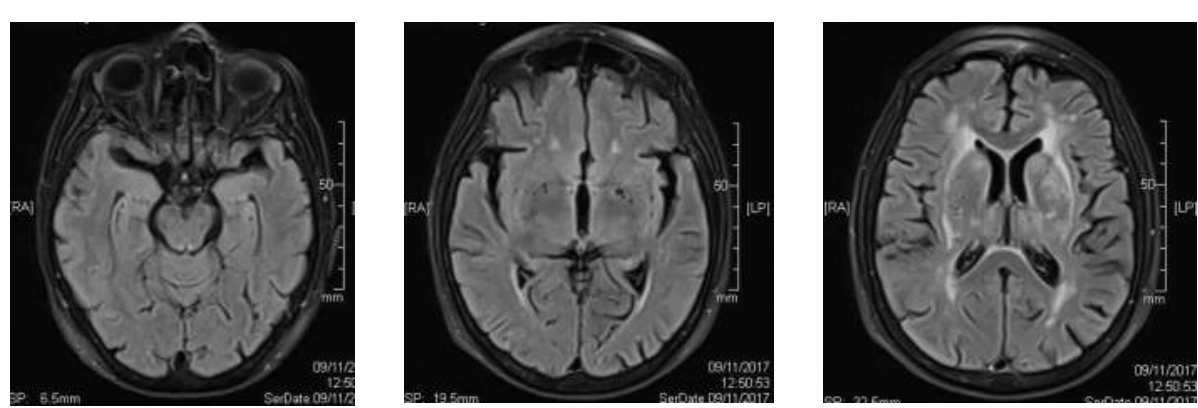

Figure 1. Patient's MRI (FLAIR images).

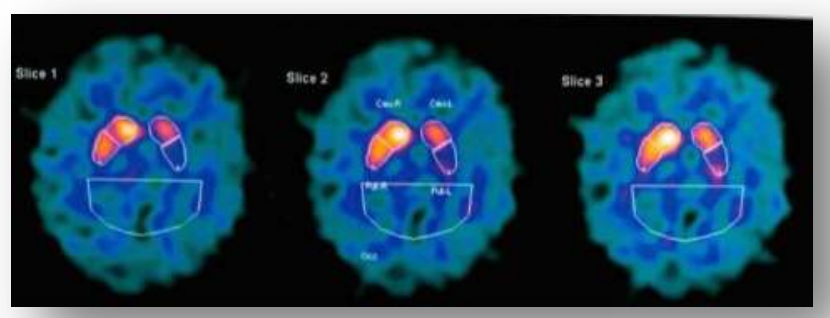

Figure 2. Patient's DaT-scan.

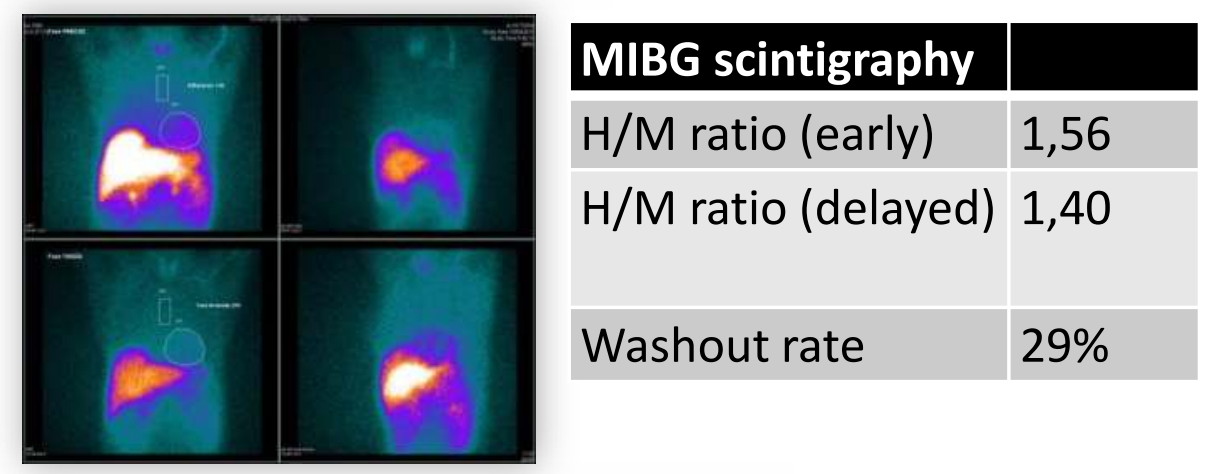

Figure 3. Patient's MIBG scintigraphy.

$\mathrm{H} / \mathrm{M}$ : heart to mediastinum

\section{Discusion and conclusions}

Parkinsonism has been rarely reported in CADASIL patients and most of them were related to VP2. This is the first case of a CADASIL patient with suspected PD instead of VP. She presented asymmetric parkinsonism, asymmetric DAT-scan alteration and good response to levodopa and dopaminergic agonists, suggesting PD. The myocardial MIBG scintigraphy was not concluent. Therefore, it is mandatory to evaluate whether a CADASIL patient with extrapyramidal symptoms could present Parkinson Disease, as an appropriate treatment could improve the quality of life of the patient.

\section{Bibliography}

1.Wegner $F$, et al. Vascular parkinsonism in a CADASIL case with an intact nigrostriatal dopaminergic system. J Neurol. 2007.

2.Ragno $\mathrm{M}$, et al. Parkinsonism is a late, not rare, feature of CADASIL a study on Italian patients carrying the R1006C mutation. Stroke. 2013.

3.Ragno M, et al. Parkinsonism in a pair of monozygotic CADASIL twins sharing the R1006C mutation: a transcranial sonography study. Neurol Sci. 2016 . 